\title{
A SIMPLE TUBE CONTROLLER FOR EFFICIENT ROBUST MODEL PREDICTIVE CONTROL OF CONSTRAINED LINEAR DISCRETE TIME SYSTEMS SUBJECT TO BOUNDED DISTURBANCES *
}

\author{
S. V. Raković ${ }^{* 1}$ D. Q. Mayne* \\ * Imperial College London, London SW7 2BT, United \\ Kingdom, Tel: +44-(0)20-7594-6295/87/81. Fax: \\ +44-(0)20-7594-6282, E-mail: sasa.rakovic@imperial.ac.uk \\ and d.mayne@imperial.ac.uk
}

\begin{abstract}
We present a simple tube controller for efficient robust model predictive control of constrained linear, discrete-time systems in the presence of bounded disturbances. The proposed control policy ensures that controlled trajectories are confined to a given tube despite uncertainty. The robust optimal control problem that is solved on-line is a standard quadratic programming problem of marginally increased complexity compared with that required for model predictive control in the deterministic case. We show how to optimize the tube cross section, how to construct an adequate tube terminal set and we establish robust exponential stability of a suitable robust control invariant set (the 'origin' for uncertain system) with enlarged domain of attraction. Copyright ${ }^{\circledR}$ 2005 IFAC.
\end{abstract}

Keywords: Invariant Tube, Robust Constrained Control, Bounded Disturbances.

\section{INTRODUCTION}

Research on robust model predictive control has recognized the advantages of feedback control when uncertainty is present (Mayne et al., 2000; Findeisen et al., 2003). The decision variable in feedback model predictive control is a control policy $\pi$ that is a sequence $\left\{\mu_{0}(\cdot), \mu_{1}(\cdot), \ldots, \mu_{N-1}(\cdot)\right\}$ of control laws. Determination of a feedback control policy is usually prohibitively difficult and various simplifying approximations have been proposed in the literature (Kouvaritakis et al., 2000; Chisci et al., 2001; Mayne and Langson, 2001; Löfberg, 2003; van Hessem and Bosgra, 2003; Kerrigan and Alamo, 2004; Smith, 2004). Both open-

\footnotetext{
* Research supported by the Engineering and Physical Sciences Research Council, UK.

1 Corresponding Author.
}

loop and feedback model predictive controller generate a tube of trajectories when uncertainty is present. Feedback model predictive control reduces the spread of predicted trajectories resulting from uncertainty. In this note we present a simple, computationally efficient, controller that forces controlled trajectories to remain within a suitably designed tube (sequence of sets of states), ensuring robust constraint satisfaction and appropriate stability properties. Tubes have been extensively studied, see for instance (Quincampoix and Veliov, 2002; Calafiore and El Ghaoui, 2004; Kurzhanski, 2004) and references therein. Application of tubes to robust model predictive control has been reported in (Langson et al., 2004; Mayne et al., 2005; Raković and Mayne, 2004).

Our main focus is the choice of tube cross-section, tube terminal set and cost function in order to 
simplify the resultant robust optimal control problem. Section 2 is concerned with the preliminaries. Section 3 discusses an appropriate choice for tube cross-section and tube terminal set. Section 4 presents a simple formulation of the resultant robust optimal control problem and discusses stability properties. Section 5 presents an illustrative numerical example. Finally, Section 6 presents conclusions.

Notation: Let $\mathbb{N} \triangleq\{0,1,2, \ldots\}, \mathbb{N}_{+} \triangleq\{1,2, \ldots\}$ and $\mathbb{N}_{q} \triangleq\{0,1, \ldots, q\}$. A polyhedron is the (convex) intersection of a finite number of open and/or closed half-spaces and a polytope is the closed and bounded polyhedron. Given two sets $\mathcal{U}$ and $\mathcal{V}$, such that $\mathcal{U} \subset \mathbb{R}^{n}$ and $\mathcal{V} \subset \mathbb{R}^{n}$, the Minkowski (vector) sum is defined by $\mathcal{U} \oplus \mathcal{V} \triangleq\{u+v \mid$ $u \in \mathcal{U}, v \in \mathcal{V}\}$, the Pontryagin (geometric) set difference is: $\mathcal{U} \ominus \mathcal{V} \triangleq\{x \mid x \oplus \mathcal{V} \subseteq \mathcal{U}\}$. Given the sequence of sets $\left\{\mathcal{U}_{i} \subset \mathbb{R}^{n}\right\}_{i=a}^{b}$, we denote $\bigoplus_{i=a}^{b} \mathcal{U}_{i} \triangleq \mathcal{U}_{a} \oplus \cdots \oplus \mathcal{U}_{b}$. We denote $d(z, X) \triangleq$ $\inf \{|z-x| \mid x \in X\}$.

\section{PRELIMINARIES}

We consider the following discrete-time linear time-invariant (DLTI) system:

$$
x^{+}=A x+B u+w,
$$

where $x \in \mathbb{R}^{n}$ is the current state, $u \in \mathbb{R}^{m}$ is the current control action $x^{+}$is the successor state, $w \in \mathbb{R}^{n}$ is an unknown disturbance and $(A, B) \in \mathbb{R}^{n \times n} \times \mathbb{R}^{n \times m}$. The disturbance $w$ is persistent, but contained in a convex and compact set $W \subset \mathbb{R}^{n}$ that contains the origin. We make the standing assumption that the couple $(A, B)$ is controllable. We also define the corresponding nominal system:

$$
z^{+}=A z+B v
$$

where $z \in \mathbb{R}^{n}$ is the current state, $v \in \mathbb{R}^{m}$ is the current control action $z^{+}$is the successor state of the nominal system. The system (2.1) is subject to the following set of hard state and control constraints:

$$
(x, u) \in \mathbb{X} \times \mathbb{U}
$$

where $\mathbb{X} \subseteq \mathbb{R}^{n}$ and $\mathbb{U} \subseteq \mathbb{R}^{m}$ are polyhedral and polytopic sets respectively and both contain the origin as an interior point. Let $\mathcal{W} \triangleq W^{N}$ denote the class of admissible disturbance sequences $\mathbf{w} \triangleq\left\{w(i) \mid i \in \mathbb{N}_{N-1}\right\}$. Let $\phi(i ; x, \pi, \mathbf{w})$ denotes the solution at time $i$ of (2.1) when the control policy is $\pi \triangleq\left\{\mu_{0}(\cdot), \mu_{1}(\cdot), \ldots, \mu_{N-1}(\cdot)\right\}$, where $\mu_{i}(\cdot)$ is the control law (mapping state to control) at time $i$, the disturbance sequence is $\mathbf{w}$ and the initial state is $x$ at time 0 . If the initial state of nominal model is $z$ at time 0 then we denote by $\bar{\phi}(k ; z, v)$ the solution to $(2.2)$ at time instant $k$, given the control sequence $\mathbf{v} \triangleq\left\{v_{0}, v_{1} \ldots v_{N-1}\right\}$.
Robust model predictive control is defined, as usual, by specifying a finite-horizon robust optimal control problem that is solved on-line. In this note, the robust optimal control problem is the determination of a simple tube, defined as a sequence $\mathbf{X} \triangleq\left\{X_{0}, X_{1}, \ldots, X_{N}\right\}$ of sets of states, and an associated control policy $\pi$ that minimize an appropriately chosen cost function and satisfy the following set of constraints, for a given initial condition $x \in \mathbb{X}$ :

$$
\begin{aligned}
x \in X_{0}, X_{i} \subseteq \mathbb{X}, X_{N} & \subseteq X_{f} \subseteq \mathbb{X}, \\
\mu_{i}(y) & \in \mathbb{U}, \forall y \in X_{i}, \\
A y+B \mu_{i}(y) \oplus W & \subseteq X_{i+1}, \quad \forall y \in X_{i}
\end{aligned}
$$

for all $i \in \mathbb{N}_{N-1} ; X_{f}$ is the terminal constraint set. In order to exploit linearity and convexity of the considered problem, we recall and generalize some preliminary results.

Definition 1. A set $\Omega \subset \mathbb{R}^{n}$ is a robust positively invariant (RPI) set for system $x^{+}=A x+w$ and constraint set $(\mathbb{X}, W)$ if $\Omega \subseteq \mathbb{X}$ and $A x+$ $w \in \Omega, \forall w \in W, \forall x \in \Omega$.

Definition 2. A set $\Omega \subset \mathbb{R}^{n}$ is a robust control invariant (RCI) set for system $x^{+}=A x+B u+w$ and constraint set $(\mathbb{X}, \mathbb{U}, W)$ if $\Omega \subseteq \mathbb{X}$ and for every $x \in \Omega$ there exists a $u \in \mathbb{U}$ such that $A x+B u+w \in \Omega, \forall w \in W$.

The definition of a positively invariant (PI) set for $x^{+}=A x$ and constraint set $\mathbb{X}$ is given by Definition 1 with $W=\{0\}$. Similarly, the definition of a control invariant (CI) set for $x^{+}=$ $A x+B u$ and constraint set $(\mathbb{X}, \mathbb{U})$ is given by Definition 2 with $W=\{0\}$. First we generalize Proposition 1 of (Mayne and Langson, 2001):

Proposition 1. Let $\Omega$ be a RCI set for (2.1) and constraint set $(\mathbb{X}, \mathbb{U}, W)$, and let $\nu: \Omega \rightarrow \mathbb{U}$ be a control law such that $\Omega$ is a RPI set for system $x^{+}=A x+B \nu(x)+w$ and constraint set $\left(\mathbb{X}_{\nu}, W\right)$, where $\mathbb{X}_{\nu} \triangleq \mathbb{X} \cap\{x \mid \nu(x) \in \mathbb{U}\}$. Let also $x \in z \oplus \Omega$ and $u=v+\nu(x-z)$. Then for all $v \in \mathbb{R}^{m}$, $x^{+} \in z^{+} \oplus \Omega$ where $x^{+} \triangleq A x+B u+w, w \in W$ and $z^{+} \triangleq A z+B v$.

This result allows us to exploit a simple parameterization of the tube-policy pair $(\mathbf{X}, \pi)$ as follows. The state tube $\mathbf{X}=\left\{X_{0}, X_{1}, \ldots, X_{N}\right\}$ is parametrized by $\left\{z_{i}\right\}$ and $\mathcal{R}$ as follows:

$$
X_{i} \triangleq z_{i} \oplus \mathcal{R}, i \in \mathbb{N}_{N}
$$

where $z_{i}$ is the tube cross-section center at time $i$ and $\mathcal{R}$ is a set. The control laws $\mu_{i}(\cdot)$ defining the control policy $\pi=\left\{\mu_{0}(\cdot), \mu_{1}(\cdot), \ldots, \mu_{N-1}(\cdot)\right\}$ are parametrized by $\left\{z_{i}\right\}$ and $\left\{v_{i}\right\}$ as follows: 


$$
\mu_{i}(y) \triangleq v_{i}+\nu\left(y-z_{i}\right), y \in X_{i},
$$

for all $i \in \mathbb{N}_{N-1}$, where $v_{i}$ is the feedforward component of the control law and $\nu\left(y-z_{i}\right)$ is feedback component of the control law $\mu_{i}(\cdot)$. A suitable choice for the tube cross-section $\mathcal{R}$ is any RCI set with a $\nu: \mathcal{R} \rightarrow \mathbb{U}$ such that $\mathcal{R}$ is RPI for system $A x+B \nu(x)+w$ and constraint set $\left(\mathbb{X}_{\nu}, W\right)$, where $\mathbb{X}_{\nu} \triangleq \mathbb{X} \cap\{x \mid \nu(x) \in \mathbb{U}\}$. The sequence $\left\{z_{i}\right\}$ is the sequence of tube centers and is required to satisfy (2.2), subject to tighter constraints than those in (2.2), as discussed in the sequel.

\section{CONSTRUCTION OF A SIMPLE TUBE}

\subsection{Optimized tube cross-section}

To reduce conservativeness we minimize an appropriate norm of the set $\mathcal{R}$ by exploiting a relevant result recently established in (Raković, 2005). Let $M_{i} \in \mathbb{R}^{m \times n}, i \in \mathbb{N}$ and for each $k \in \mathbb{N}$ let $\mathbf{M}_{k} \triangleq\left(M_{0}, M_{1}, \ldots, M_{k-2}, M_{k-1}\right)$. An appropriate characterization of a family of RCI sets for (2.1) for unconstrained case, for constraint set $\left(\mathbb{R}^{n}, \mathbb{R}^{m}, W\right)$, is given by the following sets for $k \geq n$ :

$$
R_{k}\left(\mathbf{M}_{k}\right) \triangleq \bigoplus_{i=0}^{k-1} D_{i}\left(\mathbf{M}_{k}\right) W
$$

where the matrices $D_{i}\left(\mathbf{M}_{k}\right), i \in \mathbb{N}_{k}, k \geq n$ are defined by:

$$
\begin{aligned}
& D_{0}\left(\mathbf{M}_{k}\right) \triangleq I, \\
& D_{i}\left(\mathbf{M}_{k}\right) \triangleq A^{i}+\sum_{j=0}^{i-1} A^{i-1-j} B M_{j}, i \geq 1
\end{aligned}
$$

where $\mathbf{M}_{k}$ satisfies:

$$
D_{k}\left(\mathbf{M}_{k}\right)=\mathbf{0}
$$

Since the couple $(A, B)$ is assumed to be controllable, such a choice exists for all $k \geq n$. Let $\mathbb{M}_{k}$ denote the set of all matrices $\mathbf{M}_{k}$ satisfying condition (3.3):

$$
\mathbb{M}_{k} \triangleq\left\{\mathbf{M}_{k} \mid D_{k}\left(\mathbf{M}_{k}\right)=\mathbf{0}\right\}
$$

Theorem 1. (Raković, 2005) Given any $\mathbf{M}_{k} \in \mathbb{M}_{k}$ and the corresponding set $R_{k}\left(\mathbf{M}_{k}\right)$ there exists a control law $\nu: R_{k}\left(\mathbf{M}_{k}\right) \rightarrow \mathbb{R}^{m}$ such that $A x+B \nu(x) \oplus W \subseteq R_{k}\left(\mathbf{M}_{k}\right), \forall x \in R_{k}\left(\mathbf{M}_{k}\right)$, i.e. the set $R_{k}\left(\mathbf{M}_{k}\right)$ is RCI for the system (2.1) and constraint set $\left(\mathbb{R}^{n}, \mathbb{R}^{m}, W\right)$.

The feedback control law $\nu: R_{k}\left(\mathbf{M}_{k}\right) \rightarrow \mathbb{R}^{m}$ in Theorem 1 is a selection from the set valued map:

$$
\mathcal{U}(x) \triangleq \mathbf{M}_{k} \mathbf{W}(x)
$$

where $\mathbf{M}_{k} \in \mathbb{M}_{k}$ and the set of disturbance sequences $\mathbf{W}(x)$ is defined for each $x \in R_{k}\left(\mathbf{M}_{k}\right)$ by:

$$
\mathbf{W}(x) \triangleq\left\{\mathbf{w} \mid \mathbf{w} \in \mathbf{W}^{k}, D \mathbf{w}=x\right\},
$$

where $\mathbf{W}^{k} \triangleq W \times W \times \ldots \times W$ and $D=$ $\left[D_{k-1}\left(\mathbf{M}_{k}\right) \ldots D_{0}\left(\mathbf{M}_{k}\right)\right]$. A $\nu(\cdot)$ satisfying Theorem 1 can be defined, for instance, as follows:

$$
\begin{aligned}
\nu(x) & \triangleq \mathbf{M}_{k} \mathbf{w}^{0}(x) \\
\mathbf{w}^{0}(x) & \triangleq \arg \min _{\mathbf{w}}\left\{|\mathbf{w}|^{2} \mid \mathbf{w} \in \mathbf{W}(x)\right\}
\end{aligned}
$$

The function $\mathbf{w}^{0}(\cdot)$ is piecewise affine, being the solution of a parametric quadratic programme; thus, the feedback control law $\nu: R_{k}\left(\mathbf{M}_{k}\right) \rightarrow$ $\mathbb{R}^{m}$ is piecewise affine (being a linear map of a piecewise affine function). The set $R_{k}\left(\mathbf{M}_{k}\right)$ and the feedback control law $\nu(\cdot)$ are parametrized by the matrix $\mathbf{M}_{k}$. It is shown in (Raković, 2005) that a suitable $\mathbf{M}_{k}$ can be obtained by solving an optimization problem. The optimization problem is:

$$
\begin{aligned}
\overline{\mathbb{P}}_{k}: & \left(\mathbf{M}_{k}^{0}, \alpha^{0}, \beta^{0}, \delta^{0}\right)= \\
\quad & \arg \min _{\mathbf{M}_{k}, \alpha, \beta, \delta}\left\{\delta \mid\left(\mathbf{M}_{k}, \alpha, \beta, \delta\right) \in \bar{\Omega}\right\}
\end{aligned}
$$

where the constraint set $\bar{\Omega}$ is defined by:

$$
\begin{aligned}
\bar{\Omega} \triangleq\left\{\left(\mathbf{M}_{k}, \alpha, \beta, \delta\right) \mid\right. & \mathbf{M}_{k} \in \mathbb{M}_{k}, R_{k}\left(\mathbf{M}_{k}\right) \subseteq \alpha X, \\
& U\left(\mathbf{M}_{k}\right) \subseteq \beta U, \\
& (\alpha, \beta) \in[0,1] \times[0,1] \\
& \left.q_{\alpha} \alpha+q_{\beta} \beta \leq \delta\right\}
\end{aligned}
$$

with $R_{k}\left(\mathbf{M}_{k}\right)$ defined by (3.1), $U\left(\mathbf{M}_{k}\right)$ defined by:

$$
U\left(\mathbf{M}_{k}\right) \triangleq \bigoplus_{i=0}^{k-1} M_{i} W
$$

with weights $q_{\alpha}$ and $q_{\beta}$ reflecting a desired contraction of state and control constraint sets. The solution $\mathbf{M}_{k}^{0}$ to problem $\overline{\mathbb{P}}_{k}$ (which exists if $\bar{\Omega} \neq \emptyset$ ) yields a set $R_{k}^{0} \triangleq R_{k}\left(\mathbf{M}_{k}^{0}\right)$ and feedback control law $\nu^{0}(x)=\mathbf{M}_{k}^{0} \mathbf{w}^{0}(x)$ satisfying:

$$
R_{k}^{0} \subseteq \alpha^{0} \mathbb{X}, \nu^{0}(x) \in U\left(\mathbf{M}_{k}\right) \subseteq \beta^{0} \mathbb{U},
$$

for all $x \in R_{k}^{0}$. It follows from Theorem 1 and the discussion above that the set $R_{k}^{0}$, if it exists, is RPI for system $x^{+}=A x+B \nu^{0}(x)+w$ and constraint set $\left(\mathbb{X}_{\nu^{0}}, W\right)$, where $\mathbb{X}_{\nu^{0}} \triangleq \alpha^{0} \mathbb{X} \cap\left\{x \mid \nu^{0}(x) \in\right.$ $\left.\beta^{0} \mathbb{U}\right\}$.

\subsection{Tube Terminal Set}

The parametrization for the state tube $\mathbf{X}$ motivates the introduction of a set of sets of the form $\Phi \triangleq\left\{z \oplus \mathcal{R} \mid z \in Z_{f}\right\}$ ( $\Phi$ is a set of sets, each of the form $z \oplus \mathcal{R}$ where $\mathcal{R}$ is a set) that is set robust control invariant.

Definition 3. A set of sets $\Phi$ is set robust control invariant (SRCI) for system $x^{+}=A x+B u+w$ and constraint set $(\mathbb{X}, \mathbb{U}, W)$ if for any set $X \in \Phi$ : (i) $X \subseteq \mathbb{X}$ and, (ii) there exists a set $Y \in \Phi$ such 
that for all $x \in X$, there exists a $u \in \mathbb{U}$ such that $A x+B u \oplus W \subseteq Y$.

We assume that:

A1: (i) The set $\mathcal{R}$ is a RCI set for system (2.1) and constraint set $(\alpha \mathbb{X}, \beta \mathbb{U}, W)$ where $(\alpha, \beta) \in[0,1) \times$ $[0,1)$, (ii) The control law $\nu: \mathcal{R} \rightarrow \beta \mathbb{U}$ is such that $\mathcal{R}$ is RPI for system $x^{+}=A x+B \nu(x)+w$ and constraint set $\left(\mathbb{X}_{\nu}, W\right)$, where $\mathbb{X}_{\nu} \triangleq \alpha \mathbb{X} \cap X_{\nu}$ where $X_{\nu} \triangleq\{x \mid \nu(x) \in \beta \mathbb{U}\}(\nu(\cdot)$ exists by $\mathbf{A} \mathbf{1}$ (i)).

Let $U_{\nu}, \mathbb{Z}, \mathbb{V}$ be defined as follows:

$$
U_{\nu} \triangleq\{\nu(x) \mid x \in \mathcal{R}\}, \mathbb{Z} \triangleq \mathbb{X} \ominus \mathcal{R}, \mathbb{V} \triangleq \mathbb{U} \ominus U_{\nu} .
$$

We also assume:

A2: (i) The set $Z_{f}$ is a CI set for the nominal system (2.2) and constraint set $(\mathbb{Z}, \mathbb{V})$, (ii) The control law $\varphi: Z_{f} \rightarrow \mathbb{V}$ is such that $Z_{f}$ is PI for system $z^{+}=A z+B \varphi(z)$ and constraint set $\mathbb{Z}_{\varphi}$, where $\mathbb{Z}_{\varphi} \triangleq \mathbb{Z} \cap\{z \mid \varphi(z) \in \mathbb{V}\}$. ( $\varphi(\cdot)$ exists by A2 (i)).

We can now establish the following relevant result:

Theorem 2. Suppose that $\mathbf{A} \mathbf{1}$ and $\mathbf{A} \mathbf{2}$ are satisfied. Then $\Phi \triangleq\left\{z \oplus \mathcal{R} \mid z \in Z_{f}\right\}$ is a set robust control invariant for system $x^{+}=A x+B u+w$ and constraint set $(\mathbb{X}, \mathbb{U}, W)$.

PROOF. Let $X \in \Phi$, then $X=z \oplus \mathcal{R}$ for some $z \in Z_{f}$. For every $x \in X$ we have $x=z+$ $y$, where $y \triangleq x-z \in \mathcal{R}$. Let the control law $\theta: Z_{f} \oplus \mathcal{R} \rightarrow \mathbb{U}$ be defined by $\theta(x) \triangleq \varphi(z)+\nu(y)$ and let $u=\theta(x)$. Then $x^{+}=A x+B \theta(x)+$ $w=A(z+y)+B(\varphi(z)+\nu(y))+w=A z+$ $B \varphi(z)+A y+B \nu(y)+w$. It follows from $\mathbf{A} 2$ that $A z+B \varphi(z) \in Z_{f}$ and, by $\mathbf{A} \mathbf{1}$, we have $A y+B \nu(y)+w \in \mathcal{R}, \forall(y, w) \in \mathcal{R} \times W$,. Hence we conclude that $A x+B \theta(x)+w \in Y, \forall(x, w) \in X \times$ $W$ where $Y \triangleq z^{+} \oplus \mathcal{R} \in \Phi$. The fact that $X \subseteq \mathbb{X}$ follows from A1 and A2 because $Z_{f} \oplus \mathcal{R} \subseteq \mathbb{X}$. The fact that $u=\theta(x) \in \mathbb{U}$ for all $x \in X$ and every $X \in \Phi$ follows from $\mathbf{A} \mathbf{1}$ and $\mathbf{A} \mathbf{2}$ since $\varphi(z) \in \mathbb{U} \ominus$ $U_{\nu} \subseteq(1-\beta) \mathbb{U}, \forall z \in Z_{f}$ and $\nu(y) \in \beta \mathbb{U}, \forall y \in \mathcal{R}$ implying that $u=\theta(x) \in \mathbb{U}, \forall x \in X$ and every $X \in \Phi$.

The terminal set $X_{f}$ is defined by:

$$
X_{f} \triangleq Z_{f} \oplus \mathcal{R}
$$

where the sets $\mathcal{R}$ and $Z_{f}$ satisfy $\mathbf{A} \mathbf{1}$ and $\mathbf{A} \mathbf{2}$. With this choice for terminal set the domain of attraction is enlarged (compared to the case when $\left.X_{f}=\mathcal{R}\right)$.

\section{SIMPLE TUBE CONTROLLER}

We are now ready to propose a robust optimal control problem, whose solution yields the tube and the corresponding control policy satisfying the set of constraints specified in $(2.4)-(2.6)$ providing that there exists $R C I$ set $\mathcal{R}=R_{k}^{0}=$ $R_{k}\left(\mathbf{M}_{k}^{0}\right), k \in \mathbb{N}$ (for the system $x^{+}=A x+$ $B u+w$ and constraint set $(\alpha \mathbb{X}, \beta \mathbb{U}, W)$, with $(\alpha, \beta) \in[0,1) \times[0,1))$ defined in (3.1) with the corresponding feedback control law $(3.6)-(3.7)$. We define:

$$
\mathbb{Z} \triangleq \mathbb{X} \ominus \mathcal{R}, \mathbb{V} \triangleq \mathbb{U} \ominus U\left(\mathbf{M}_{k}^{0}\right)
$$

where $\mathcal{R} \triangleq R_{k}^{0}=R_{k}\left(\mathbf{M}_{k}^{0}\right), R_{k}\left(\mathbf{M}_{k}^{0}\right)$ and $U\left(\mathbf{M}_{k}^{0}\right)$ are defined in (3.1) and (3.10), respectively. In order to insure satisfaction of $(2.4)-(2.6)$ and use of the simple tube-policy parametrization (2.7) (2.8) we require that the trajectory of the nominal model (the sequence of tube centers) satisfies the tighter constraints (4.1). We assume in the sequel that $\mathbf{A} \mathbf{1}$ and $\mathbf{A} \mathbf{2}$ hold so that the terminal set $Z_{f}$ for the nominal model can be any CI set satisfying A2. Let the set $\mathcal{V}_{N}(x)$ of admissible control-states pairs for nominal system at state $x$ be defined as follows:

$$
\begin{array}{r}
\mathcal{V}_{N}(x) \triangleq\left\{(\mathbf{v}, z) \mid\left(\bar{\phi}(k ; z, \mathbf{v}), v_{k}\right) \in \mathbb{Z} \times \mathbb{V}\right. \\
\left.k \in \mathbb{N}_{N-1}, \bar{\phi}(N ; z, \mathbf{v}) \in Z_{f}, x \in z \oplus \mathcal{R}\right\}
\end{array}
$$

It is clear that the set $\mathcal{V}_{N}(x)$ is a polyhedral set providing that $Z_{f}$ is a polyhedral set. An appropriate cost function can be defined as follows:

$$
V_{N}(\mathbf{v}, z) \triangleq \sum_{i=0}^{N-1} \ell\left(z_{i}, v_{i}\right)+V_{f}\left(z_{N}\right),
$$

where for all $i, z_{i} \triangleq \bar{\phi}(i ; z, \mathbf{v})$ and $\ell(\cdot)$ is the stage cost and $V_{f}(\cdot)$ is the terminal cost, that can be chosen to be :

$$
\begin{aligned}
\ell(x, u) & \triangleq\|Q x\|_{p}+\|R u\|_{p}, p=1,2, \infty \\
V_{f}(x) & \triangleq\|P x\|_{p}, p=1,2, \infty
\end{aligned}
$$

where $P, Q$ and $R$ are matrices of suitable dimensions. We assume additionally, as is standard (Mayne et al., 2000), that:

A3: The terminal cost satisfies $V_{f}(A z+B \varphi(z))+$ $\ell(z, \varphi(z)) \leq V_{f}(z)$ for all $z \in Z_{f}$.

If $p=2$ and $Q=Q^{\prime}>0, P=P^{\prime}>0$ and $R=R^{\prime}>0$ the resultant optimal control problem is a quadratic programme (Mayne et al., 2005), since the set $\mathcal{V}_{N}(x)$ is polyhedral, defined by :

$\mathbb{P}_{N}(x): \quad V_{N}^{0}(x) \triangleq \min _{\mathbf{v}, z}\left\{V_{N}(\mathbf{v}, z) \mid(\mathbf{v}, z) \in \mathcal{V}_{N}(x)\right\}$

and its unique minimizer is:

$\left(\mathbf{v}^{0}(x), z^{0}(x)\right) \triangleq \arg \min _{\mathbf{v}, z}\left\{V_{N}(\mathbf{v}, z) \mid(\mathbf{v}, z) \in \mathcal{V}_{N}(x)\right\}$

The domain of the value function $V_{N}^{0}(\cdot)$, the controllability set, is: 


$$
\mathcal{X}_{N} \triangleq\left\{x \mid \mathcal{V}_{N}(x) \neq \emptyset\right\}
$$

For each $i$ let $\mathcal{V}_{i}(x)$ and $\mathcal{X}_{i}$ be defined, respectively, by (4.2) and (4.7) with $i$ replacing $N$. The sequence $\left\{\mathcal{X}_{i}\right\}$ is a monotonically non-decreasing set sequence, i.e. $\mathcal{X}_{i} \subseteq \mathcal{X}_{i+1}$ for all $i$. Given any $x \in \mathcal{X}_{N}$ the solution to $\mathbb{P}_{N}(x)$ defines the corresponding optimal simple tube:

$$
\mathbf{X}^{0}(x)=\left\{X_{i}^{0}(x)\right\}, X_{i}^{0}(x)=z_{i}^{0}(x) \oplus \mathcal{R},
$$

for $i \in \mathbb{N}_{N}$, and the corresponding control policy $\pi^{0}(x)=\left\{\mu_{i}^{0}(\cdot) \mid i \in \mathbb{N}_{N-1}\right\}$ with

$$
\mu_{i}^{0}(y ; x)=v_{i}^{0}(x)+\nu\left(y-z_{i}^{0}(x)\right), y \in X_{i}^{0}(x)
$$

where, for each $i, z_{i}^{0}(x)=\bar{\phi}\left(i ; z^{0}(x), \mathbf{v}^{0}(x)\right)$. The following result (Langson et al., 2004) follows easily from the construction of a simple tube:

Proposition 2. Let $x \in \mathcal{X}_{N}$ and let $\left(\mathbf{v}^{0}(x), z^{0}(x)\right)$ be defined by (4.6). Let the control policy $\pi^{0} \triangleq$ $\left\{\mu_{i}^{0}(\cdot) \mid i \in \mathbb{N}_{N-1}\right\}$, where each $\mu_{i}^{0}: X_{i} \rightarrow \mathbb{U}$ is defined by (4.9), be applied to actual system (2.1). Then $\phi(i ; x, \pi, \mathbf{w}) \in \bar{\phi}\left(i ; z^{0}(x), \mathbf{v}^{0}(x)\right) \oplus$ $\mathcal{R}$ for all $i \in \mathbb{N}_{N}$ and all $\mathbf{w} \in \mathcal{W}$. Moreover, $\left(\phi\left(i ; x, \pi^{0}, \mathbf{w}\right), \mu_{i}^{0}\left(\phi\left(i ; x, \pi^{0}, \mathbf{w}\right) ; x\right)\right) \in \mathbb{X} \times \mathbb{U}, i \in$ $\mathbb{N}_{N-1}$ and $\phi\left(N ; x, \pi^{0}, \mathbf{w}\right) \in X_{f}$.

A result analogous to Proposition 2 holds for any arbitrary couple $(\mathbf{v}, z) \in \mathcal{V}_{N}(x)$. The solution of $\mathbb{P}_{N}(x)$ allows for a variety of controller implementations. Here we follow a useful proposal recently made in (Mayne et al., 2005) and consider the following implicit robust model predictive control law $\kappa_{N}^{0}(\cdot)$ yielded by the solution of $\mathbb{P}_{N}(x)$ :

$$
\kappa_{N}^{0}(x) \triangleq v_{0}^{0}(x)+\nu\left(x-z^{0}(x)\right)
$$

where $\nu(\cdot)$ is defined in (3.6) - (3.7). We establish some relevant properties of the proposed controller $\kappa_{N}^{0}(\cdot)$ by exploiting the results reported in (Mayne et al., 2005). First we recall the following definition:

Definition 4. A set $\mathcal{R}$ is robustly exponentially stable (Lyapunov stable and exponentially attractive) for $x^{+}=A x+B \kappa(x)+w, w \in W$, with a region of attraction $\mathcal{X}$ if there exists a $c>0$ and a $\gamma \in(0,1)$ such that any solution $x(\cdot)$ of $x^{+}=$ $A x+B \kappa(x)+w$ with initial state $x(0) \in \mathcal{X}$, and admissible disturbance sequence $w(\cdot)(w(i) \in W$ for all $i \geq 0)$ satisfies $d(x(i), \mathcal{R}) \leq c \gamma^{i} d(x(0), \mathcal{R})$ for all $i \geq 0$.

Proposition 3. (i) For all $x \in \mathcal{R}, V_{N}^{0}(x)=0$, $z^{0}(x)=0, \mathbf{v}^{0}(x)=\{0,0, \ldots, 0\}$ and $\kappa_{N}^{0}(x)=$ $\nu(x)$. (ii) Let $x \in \mathcal{X}_{N}$ and let $\left(\mathbf{v}^{0}(x), z^{0}(x)\right)$ be defined by (4.6), then for all $x^{+} \in A x+B \kappa_{N}^{0}(x) \oplus$ $W$ there exists $\left(\mathbf{v}\left(x^{+}\right), z\left(x^{+}\right)\right) \in \mathcal{V}_{N}\left(x^{+}\right)$and

$$
V_{N}^{0}\left(x^{+}\right) \leq V_{N}^{0}(x)-\ell\left(z^{0}(x), v_{0}^{0}(x)\right) .
$$

The main stability result follows (see Theorem 1 in (Mayne et al., 2005)):

Theorem 3. Suppose that $\mathcal{X}_{N}$ is bounded, then the set $\mathcal{R}$ is robustly exponentially stable for controlled uncertain system $x^{+}=A x+B \kappa_{N}^{0}(x)+$ $w, w \in W$. The region of attraction is $\mathcal{X}_{N}$.

The proposed controller $\kappa_{N}^{0}(\cdot)$ results in a set sequence $\left\{X_{0}^{0}(x(i))\right\}$, where:

$$
X_{0}^{0}(x(i))=z^{0}(x(i)) \oplus \mathcal{R}, i \in \mathbb{N}
$$

and $z^{0}(x(i)) \rightarrow 0$ exponentially as $i \rightarrow \infty$. The actual trajectory $x(\cdot) \triangleq\{x(i)\}$, where $x(i)$ is the solution of $x^{+}=A x+B \kappa_{N}^{0}(x)+w$ at time $i \geq 0$, corresponding to a particular realization of an infinite admissible disturbance sequence $w(\cdot) \triangleq\left\{w_{i}\right\}$, satisfies $x(i) \in X_{0}^{0}(x(i)), \forall i \in \mathbb{N}$. Theorem 3 implies that $X_{0}^{0}(x(i)) \rightarrow \mathcal{R}$ as $i \rightarrow \infty$ exponentially in the Hausdorff metric.

\section{NUMERICAL EXAMPLE}

Our illustrative example is a double integrator:

$$
x^{+}=\left[\begin{array}{ll}
1 & 1 \\
0 & 1
\end{array}\right] x+\left[\begin{array}{l}
1 \\
1
\end{array}\right] u+w
$$

with $w \in W \triangleq\left\{\left.w \in \mathbb{R}^{2}|| w\right|_{\infty} \leq 0.5\right\}, x \in X=$ $\left\{x \in \mathbb{R}^{2} \mid x^{1} \leq 1.85, x^{2} \leq 2\right\}$ and $u \in U=$ $\{u|| u \mid \leq 2.4\}$, where $x^{i}$ is the $i^{\text {th }}$ coordinate of a vector $x$. The cost function is defined by (4.4) with $Q=100 I, R=100$; the terminal cost $V_{f}(x)$ is the value function $(1 / 2) x^{\prime} P_{f} x$ for the optimal unconstrained problem for the nominal system. The horizon is $N=21$. The design parameters for the minimization problem $\overline{\mathbb{P}}_{k}$ (See (3.10)) defining the components of feedback actions of control policy are $k=5, q_{\alpha}=q_{\beta}=1$. The optimization problem $\overline{\mathbb{P}}_{k}$, which in this case is a linear program, yielded the following matrix $\mathbf{M}_{k}^{0}$ :

$$
\mathbf{M}_{k}^{0}=\left[\begin{array}{ccccc}
-0.3833 & 0 & 0.15 & 0.233 & 0 \\
-1 & 0 & 0 & 0 & 0
\end{array}\right]^{\prime}
$$

The tube cross-section is constructed by using the set $\mathcal{R}=R_{k}\left(\mathbf{M}_{k}^{0}\right)$. The sequence of the sets $\mathcal{X}_{i}, i=0,1, \ldots, 21$, where $\mathcal{X}_{i}$ is the domain of $V_{i}^{0}(\cdot)$ and the terminal set $X_{f}=Z_{f} \oplus \mathcal{R}$ where $Z_{f}$ satisfies $\mathbf{A 2}$ and is the maximal positively invariant set (Blanchini, 1999) for system $z^{+}=(A+$ $B K) z$ under the tighter constraints $\mathbb{Z}=\mathbb{X} \ominus \mathcal{R}$ and $\mathbb{V}=\mathbb{U} \ominus U\left(\mathbf{M}_{k}^{0}\right)$ where $K$ is unconstrained DLQR controller for $(A, B, Q, R)$, is shown in Figure 1. A RMPC tube $\left\{X_{0}^{0}(x(i))=z_{0}^{0}(x(i)) \oplus \mathcal{R}\right\}$ for initial state $x_{0}=(0.5,-8.5)^{\prime}$ is shown in Figure 2 for a sequence of random admissible disturbances. The dash-dot line is the actual trajectory $\{x(i)\}$ due to the disturbance realization while the dotted line is the sequence $\left\{z_{0}^{0}(x(i))\right\}$ of optimal initial states for corresponding nominal system. 


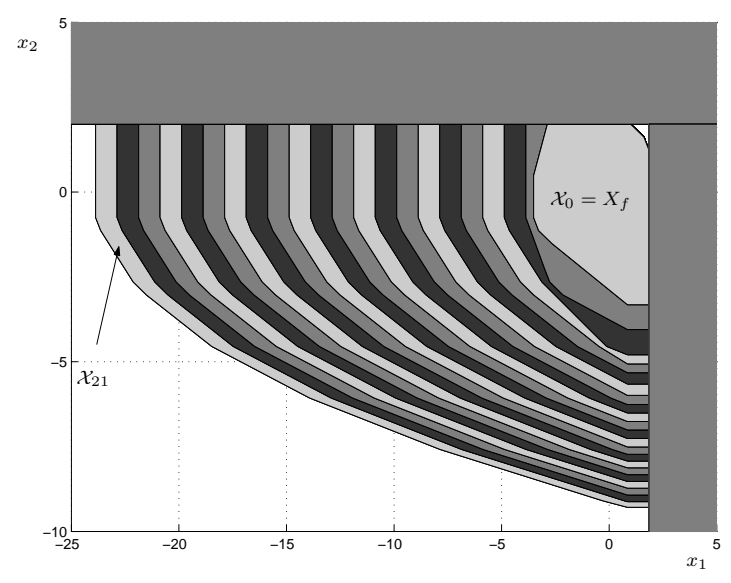

Fig. 1. Controllability Sets $\mathcal{X}_{i}, i=0,1, \ldots, 21$

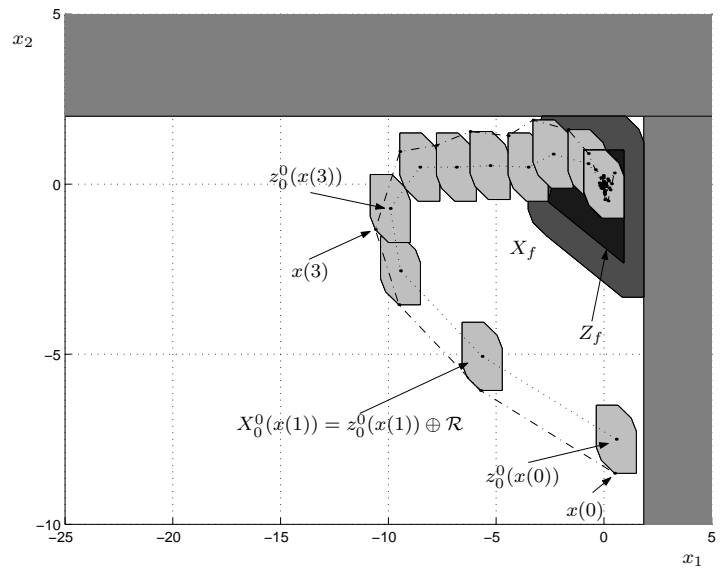

Fig. 2. RMPC Tube Trajectory

\section{CONCLUSIONS}

The main contribution of this note is a simple tube controller that ensures robust exponential stability of $\mathcal{R}$, a RCI set - the 'origin' for the uncertain system. The complexity of the corresponding robust optimal control problem is marginally increased compared with that for conventional model predictive control. A set of necessary ingredients ensuring robust exponential stability has been identified. The proposed scheme is computationally simpler than the schemes proposed in (Löfberg, 2003; van Hessem and Bosgra, 2003; Kerrigan and Alamo, 2004; Langson et al., 2004) and it has an advantage over schemes proposed in (Kouvaritakis et al., 2000; Chisci et al., 2001; Mayne and Langson, 2001; Smith, 2004; Mayne et al., 2005) because the feedback component of control policy, which is piecewise affine, results in a smaller tube cross-section.

\section{REFERENCES}

Blanchini, F. (1999). Set invariance in control. Automatica 35, 1747-1767. survey paper.

Calafiore, G. and L. El Ghaoui (2004). Ellipsoidal bounds for uncertain linear equations and dynamical systems. Automatica 40(5), 773787.

Chisci, L., J.A. Rossiter and G. Zappa (2001). Systems with persistent disturbances: predictive control with restricted constraints. Automatica 37, 1019-1028.

Findeisen, R., L. Imsland, F. Allgöwer and B. A. Foss (2003). State and output feedback nonlinear model predictive control: An overview. European Journal of Control 9(2-3), 190-206. Survey paper.

Kerrigan, E.C. and T. Alamo (2004). A convex parametrization for solving constrained minmax problems with a quadratic cost.. In: Proc. American Control Conference. Boston, MA, USA.

Kouvaritakis, B., J. A. Rossiter and J. Schuurmans (2000). Efficient robust predictive control. IEEE Trans. Automatic Control 45(8), 1545-1549.

Kurzhanski, A. B. (2004). Dynamic optimization for nonlinear target control synthesis. In: Proc. 6th IFAC Symposium - NOLCOS2004. Stuttgart, Germany.

Langson, W., I. Chryssochoos, S. V. Raković and D. Q. Mayne (2004). Robust model predictive control using tubes. Automatica 40, 125-133.

Löfberg, J. (2003). Approximations of closed-loop minimax mpc. In: Proc. IEEE CDC. pp. 1438 $-1442$.

Mayne, D. Q. and W. Langson (2001). Robustifying model predictive control of constrained linear systems. Electronics Letters 37, 14221423.

Mayne, D. Q., J. B. Rawlings, C. V. Rao and P. O. M. Scokaert (2000). Constrained model predictive control: Stability and optimality. Automatica 36, 789-814. Survey paper.

Mayne, D. Q., M. Seron and S. V. Raković (2005). Robust model predictive control of constrained linear systems with bounded disturbances. Automatica 41, 219-224.

Quincampoix, M. and V. M. Veliov (2002). Solution tubes to differential inclusions within a collection of sets. Control and Cybernetics.

Raković, S. V. and D. Q. Mayne (2004). Robust model predictive control of constrained piecewise affine discrete time systems. In: Proc. 6th IFAC Symposium - NOLCOS2004. Stuttgart, Germany.

Raković, Saša V. (2005). Robust Control of Constrained Discrete Time Systems: Characterization and Implementation. PhD thesis. Imperial College London, London, United Kingdom.

Smith, R.S. (2004). Robust model predictive control of constrained linear systems. In: Proc. American Control Conference. pp. 245 - 250.

van Hessem, D.H. and O.H. Bosgra (2003). A full solution to the constrained stochastic closedloop mpc problem via state and innovations feedback and its receding horizon implementation. In: Proc. IEEE CDC. pp. 929 - 934. 